\title{
Relaxation of a Strained Elastic Film on a Viscous Layer
}

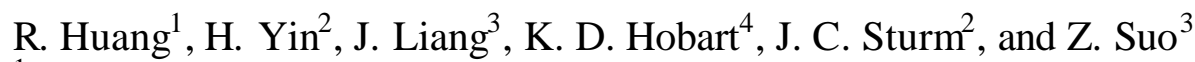

${ }^{1}$ Department of Civil and Environmental Engineering, Princeton University, Princeton, NJ 08544

${ }^{2}$ Department of Electrical Engineering and Center for Photonics \& Optoelectronic Materials, Princeton University, Princeton, NJ 08544

${ }^{3}$ Department of Mechanical \& Aerospace Engineering and Princeton Materials Institute, Princeton University, Princeton, NJ 08544

${ }^{4}$ Naval Research Laboratory, Washington, DC 20375

\section{ABSTRACT}

Experiments were conducted with SiGe film islands on a layer of borophosphorosilicate glass (BPSG). Initially the SiGe is under compression. Upon annealing, the glass flows and the SiGe islands relax by both inplane expansion and wrinkling. This paper provides a twodimensional (2D) model for inplane expansion. The results from the model are compared with the experiments with small SiGe islands. The effect of winkling, which is ignored in the present model, is discussed qualitatively.

\section{INTRODUCTION}

Various types of compliant substrates have been proposed and fabricated to grow relaxed heteroepitaxial films with low dislocation density for optoelectronic applications [1]. Figure 1 shows the schematic of one fabrication process [2]. A compressively strained SiGe film on a $\mathrm{Si}$ wafer is bonded to another Si wafer covered with a layer of borophosphorosilicate glass (BPSG), with SiGe facing BPSG. The SiGe film is very thin to avert threading dislocations. Subsequently remove the $\mathrm{Si}$ on top of $\mathrm{SiGe}$, and pattern the $\mathrm{SiGe}$ film into islands. At this stage, the $\mathrm{SiGe}$ islands remain strained. Upon annealing above the glass transition temperature, the BPSG flows and the SiGe islands relax. The goal is to obtain flat, strain-free islands, on which one can grow epitaxial optoelectronic devices. Large islands are desired; the large an island is, the more devices it can accommodate.

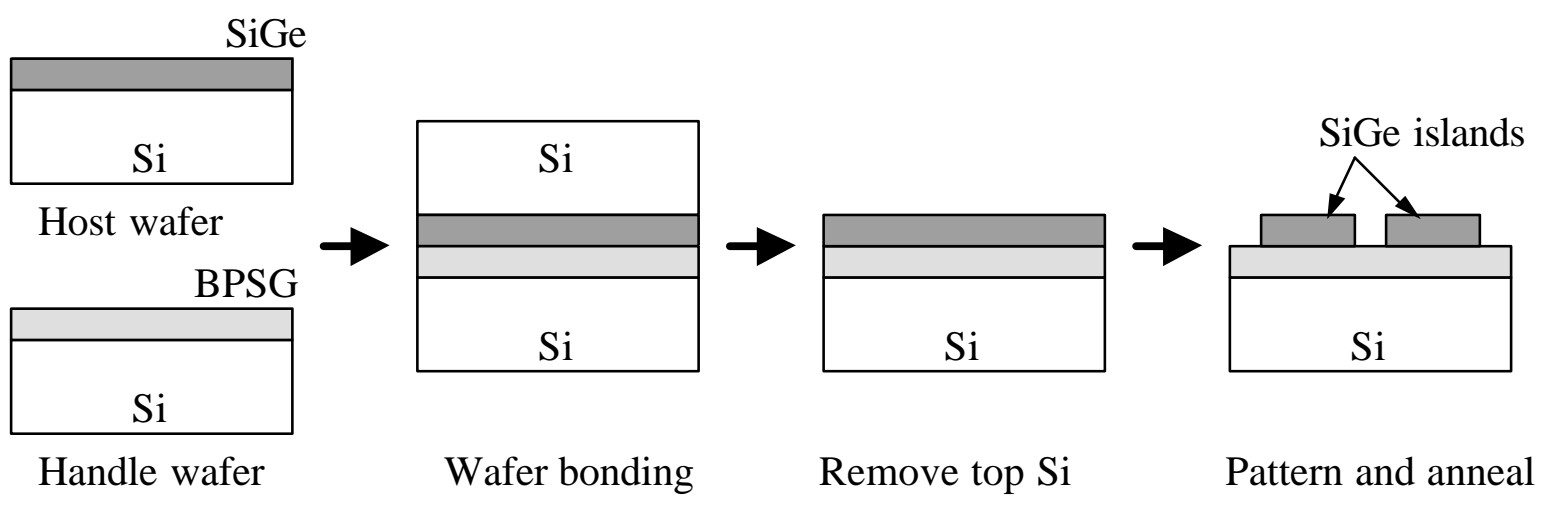

Figure 1. Schematic fabrication process 
Different relaxation mechanisms have been observed in experiments. A small island relaxes by inplane expansion. A large island wrinkles at the center. Further annealing of the large island gives rise to one of two outcomes. The wrinkles may disappear when inplane expansion arrives, leading to a flat and strain-free island. Alternatively, the wrinkles may cause significant tensile stress at the island surface, leading to fracture. Everything else being equal, a critical island size exists, below which the island relaxes without wrinkle-fracture.

Several theoretical studies have followed up the above experiments to model the relaxation process [3-8]. In this paper, we focus on relaxation of small islands, where wrinkling is not significant. We formulate a 2D model for inplane expansion of a strained film island on a viscous layer. Our approach follows that of a one-dimensional analysis by Freund and Nix [3]. The model is valid for films under either compression or tension. The effect of wrinkling is neglected, but will be discussed qualitatively.

\section{EXPERIMENT}

A wafer with a pseudomorphic $\mathrm{Si}_{\mathrm{b} .} \mathrm{Ge}_{0.3}$ film on borophosphorosilicate glass (BPSG) was fabricated using a "host" wafer and a "handle" wafer [2]. Figure 1 shows the schematic processes. The "host" wafer was a silicon wafer deposited with $30 \mathrm{~nm}$ commensurately strained $\mathrm{Si}_{0.7} \mathrm{Ge}_{0.3}$ followed by $2 \mathrm{~nm}$ Si cap, and the "handle" wafer was coated with $200 \mathrm{~nm}$ BPSG (4.4\% $\mathrm{B}$ and $4.1 \% \mathrm{P})$. The "host" wafer was implanted with $\mathrm{H}_{2}{ }^{+}$at an ion energy of $180 \mathrm{keV}$ and a dose of $4.5 \times 10^{16} \mathrm{~cm}^{-2}$. Both wafers were cleaned using $\mathrm{CO}_{2}$ snow jet to remove dusts and $\mathrm{UV}$ ozone to remove organic residue. They were then turned to hydrophilic in $\mathrm{NH}_{4} \mathrm{OH}: \mathrm{H}_{2} \mathrm{O}_{2}: \mathrm{H}_{2} \mathrm{O}:: 1: 1: 4$ and $\mathrm{HCl}: \mathrm{H}_{2} \mathrm{O}_{2}: \mathrm{H}_{2} \mathrm{O}:: 1: 1: 4$ and bonded at room temperature. The bonded wafer was annealed at $250^{\circ} \mathrm{C}$ for four hours to enhance bond strength and then at $550^{\circ} \mathrm{C}$ in $\mathrm{N}_{2}$ ambient to separate the host substrate at the $\mathrm{H}_{2}{ }^{+}$range [9]. The remaining wafer had a stack of Si/SiGe/Si/BPSG on top and was dipped into $\mathrm{KOH}$ solution $\left(10 \%\right.$ by weight) at $80^{\circ} \mathrm{C}$ to selectiviely remove the approximately $800 \mathrm{~nm} \mathrm{Si}$ on top. The wafer was diced into samples of $0.5 \mathrm{~cm}^{2}$ and the continuous $\mathrm{SiGe}$ film was patterned into arrays of square islands of various sizes by RIE etch. The samples were then annealed at $800^{\circ} \mathrm{C}$ to allow the islands to relax.

High-resolution x-ray diffraction (XRD) on (004) plane was used to determine the average lattice constant of the SiGe film in the perpendicular plane. Before annealing, the constant is $5.5308 \AA$. After annealing for 17 and 90 minutes, the measured lattice constants are measured for SiGe islands of various sizes $(\mathrm{L}=10 \mu \mathrm{m}, 20 \mu \mathrm{m}, 30 \mu \mathrm{m}$, and $40 \mu \mathrm{m})$. Wrinkling broadened and even completely washed out the XRD peak for the bigger islands $(\mathrm{L}=30 \mu \mathrm{m}$ and $40 \mu \mathrm{m})$ after 90 minutes. For the same reason, no data could be measured for islands bigger than $40 \mu \mathrm{m} \times 40 \mu \mathrm{m}$. For the cases where the lattice constant can be determined, the SiGe islands relax by inplane expansion with no significant wrinkling. The amount of relaxation is measured by the average strain in the thickness direction, as a function of the anneal time $t$, namely

$$
\bar{\varepsilon}_{\mathrm{zz}}(\mathrm{t})=\frac{\mathrm{a}_{\mathrm{z}}(\mathrm{t})-\mathrm{a}_{0}}{\mathrm{a}_{0}},
$$

where $a_{z}$ is the measured average lattice constant, $a_{0}$ is the lattice constant of a fully relaxed film. Before annealing $(\mathrm{t}=0)$, the strain is positive due to inplane compression. As the film relaxes, the strain decreases towards zero. The island of length $\mathrm{L}=10 \mu \mathrm{m}$ is considered fully relaxed after

annealing for 90 minutes, from which we obtain the fully relaxed lattice constant, $\mathrm{a}_{0}=5.4868 \AA$. 
The average strain obtained from the measured data of lattice constants through Eq. (1) will be compared to the numerical result from the $2 \mathrm{D}$ model.

\section{TWO-DIMENSIONAL MODEL}

Consider a strained elastic film of thickness $h_{f}$ bonded to a viscous layer of thickness $h_{g}$, which is in turn bonded to a rigid substrate, as shown in Fig. 2. The film is a square of side length L. Let the coordinate plane ( $x, y)$ coincides with the surface of the film, and the coordinate $\mathrm{z}$ normal to the surface. The origin of the coordinates coincides with the center of the film surface. The film is assumed to remain flat during relaxation. The film island is subject to the inplane stresses, $\sigma_{\mathrm{xx}}, \sigma_{\mathrm{yy}}$, and $\sigma_{\mathrm{xy}}$, and the shear tractions, $\tau_{\mathrm{x}}$ and $\tau_{\mathrm{y}}$, at the film/glass interface $(\mathrm{z}$ $=-\mathrm{h}_{\mathrm{f}}$ ). The equilibrium of the film island requires that

$$
\frac{\partial \sigma_{x x}}{\partial x}+\frac{\partial \sigma_{x y}}{\partial y}=\frac{\tau_{x}}{h_{f}}, \quad \frac{\partial \sigma_{x y}}{\partial x}+\frac{\partial \sigma_{y y}}{\partial y}=\frac{\tau_{y}}{h_{f}} .
$$

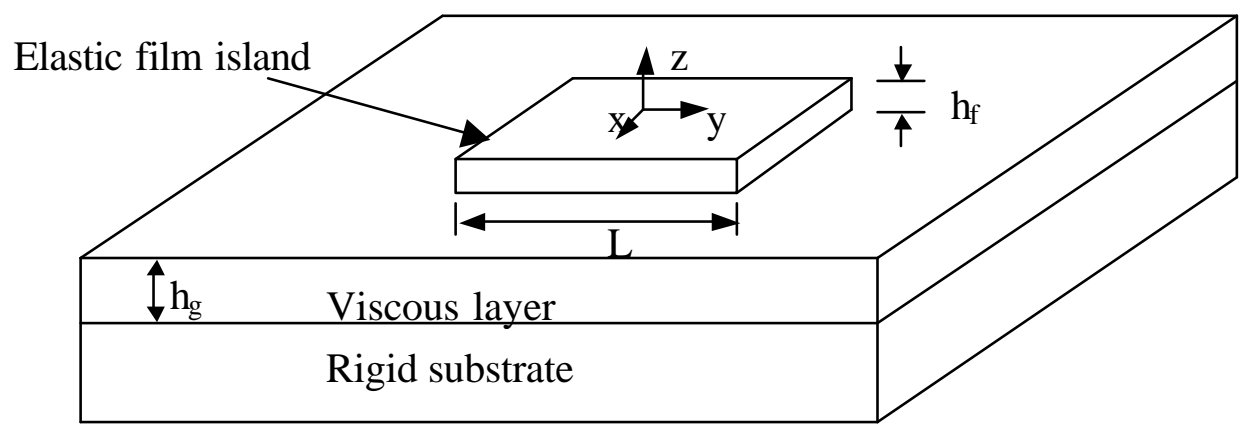

Figure 2. Illustration of a square elastic film island on a viscous layer

Assuming that $\sigma_{\mathrm{zz}}=0$ and the material is of cubic crystal symmetry, we have the thickness strain, $\varepsilon_{\mathrm{zz}}$, in terms of the inplane strain components

$$
\varepsilon_{\mathrm{zz}}=-\frac{\mathrm{c}_{12}}{\mathrm{c}_{11}}\left(\varepsilon_{\mathrm{xx}}+\varepsilon_{\mathrm{yy}}\right)
$$

By substituting Eq. (3) into the elastic stress-strain relations, we obtain that

$$
\sigma_{\mathrm{xx}}=\hat{\mathrm{c}}_{11} \varepsilon_{\mathrm{xx}}+\hat{\mathrm{c}}_{12} \varepsilon_{\mathrm{yy}}, \quad \sigma_{\mathrm{yy}}=\hat{\mathrm{c}}_{12} \varepsilon_{\mathrm{xx}}+\hat{\mathrm{c}}_{11} \varepsilon_{\mathrm{yy}}, \quad \sigma_{\mathrm{xy}}=2 \mathrm{c}_{44} \varepsilon_{\mathrm{xy}}
$$

where $\hat{\mathrm{c}}_{11}=\mathrm{c}_{11}-\mathrm{c}_{12}^{2} / \mathrm{c}_{11}$ and $\hat{\mathrm{c}}_{12}=\mathrm{c}_{12}-\mathrm{c}_{12}^{2} / \mathrm{c}_{11}$.

Before relaxation, the film is compressed with a biaxial strain, $-\varepsilon_{\mathrm{m}}$, and the displacements are zero. During relaxation, the inplane strain components are

$$
\varepsilon_{\mathrm{xx}}=\frac{\partial \mathrm{u}}{\partial \mathrm{x}}-\varepsilon_{\mathrm{m}}, \quad \varepsilon_{\mathrm{yy}}=\frac{\partial \mathrm{v}}{\partial \mathrm{y}}-\varepsilon_{\mathrm{m}}, \quad \varepsilon_{\mathrm{xy}}=\frac{1}{2}\left(\frac{\partial \mathrm{u}}{\partial \mathrm{y}}+\frac{\partial \mathrm{v}}{\partial \mathrm{x}}\right) .
$$

where $\mathrm{u}$ and $\mathrm{v}$ are the displacements in $\mathrm{x}$ and $\mathrm{y}$ directions, respectively.

\section{L3.14.3}


The glass layer is subject to the shear stresses, $\tau_{\mathrm{x}}$ and $\tau_{\mathrm{y}}$, at the top surface and is fixed at the bottom. The glass is assumed to be linearly viscous, and the flow velocity is taken to be linear from the bottom to the top surface. Assume that the displacements at the top surface of the glass are identical to the displacements of the film. Consequently, the shear stresses are

$$
\tau_{\mathrm{x}}=\frac{\eta}{\mathrm{h}_{\mathrm{g}}} \frac{\partial \mathrm{u}}{\partial \mathrm{t}}, \quad \tau_{\mathrm{y}}=\frac{\eta}{\mathrm{h}_{\mathrm{g}}} \frac{\partial \mathrm{v}}{\partial \mathrm{t}}
$$

where $\eta$ is the viscosity of the glass.

Substitution of Eqs. (4), (5), and (6) into Eq. (2) leads to

$$
\begin{aligned}
& \frac{\partial \mathrm{u}}{\partial \mathrm{t}}=\mathrm{D}\left[\overline{\mathrm{c}}_{11} \frac{\partial^{2} \mathrm{u}}{\partial \mathrm{x}^{2}}+\overline{\mathrm{c}}_{44} \frac{\partial^{2} \mathrm{u}}{\partial \mathrm{y}^{2}}+\left(\overline{\mathrm{c}}_{12}+\overline{\mathrm{c}}_{44}\right) \frac{\partial^{2} \mathrm{v}}{\partial \mathrm{x} \partial \mathrm{y}}\right], \\
& \frac{\partial \mathrm{v}}{\partial \mathrm{t}}=\mathrm{D}\left[\overline{\mathrm{c}}_{11} \frac{\partial^{2} \mathrm{v}}{\partial \mathrm{y}^{2}}+\overline{\mathrm{c}}_{44} \frac{\partial^{2} \mathrm{v}}{\partial \mathrm{x}^{2}}+\left(\overline{\mathrm{c}}_{12}+\overline{\mathrm{c}}_{44}\right) \frac{\partial^{2} \mathrm{u}}{\partial \mathrm{x} \partial \mathrm{y}}\right],
\end{aligned}
$$

where

$$
\mathrm{D}=\frac{\mathrm{c}_{11} \mathrm{~h}_{\mathrm{f}} \mathrm{h}_{\mathrm{g}}}{\eta}
$$

and $\overline{\mathrm{c}}_{11}=\hat{\mathrm{c}}_{11} / \mathrm{c}_{11}, \overline{\mathrm{c}}_{12}=\hat{\mathrm{c}}_{12} / \mathrm{c}_{11}$, and $\overline{\mathrm{c}}_{44}=\mathrm{c}_{44} / \mathrm{c}_{11}$. The quantity $\mathrm{D}$ has the dimension of diffusivity, and Eqs. (7a) and (7b) have the form of diffusion equations. The island size L and the coefficient $\mathrm{D}$ define a time scale, namely

$$
\mathrm{t}_{\mathrm{E}}=\frac{\mathrm{L}^{2}}{\mathrm{D}}=\frac{\eta \mathrm{L}^{2}}{\mathrm{c}_{11} \mathrm{~h}_{\mathrm{f}} \mathrm{h}_{\mathrm{g}}}
$$

At the edge of the film island, we have the traction-free conditions, i.e., $\sigma_{x x}(x= \pm L / 2)=$ $\sigma_{\mathrm{xy}}(\mathrm{x}= \pm \mathrm{L} / 2)=0$ and $\sigma_{\mathrm{yy}}(\mathrm{y}= \pm \mathrm{L} / 2)=\sigma_{\mathrm{xy}}(\mathrm{y}= \pm \mathrm{L} / 2)=0$. Equations (7a) and (7b) are solved numerically, and the results are discussed in the next section.

\section{RESULTS}

After normalizing Eq. (7) and the boundary conditions with the length $\mathrm{L}$ and the time $\mathrm{t}_{\mathrm{E}}$, the problem becomes independent of $\mathrm{D}$ or $\mathrm{L}$. We solve the problem by using the finite difference method. The mismatch strain is taken to be $\varepsilon_{\mathrm{m}}=1.2 \%$ for the $\mathrm{Si}_{0.7} \mathrm{Ge}_{0.3}$ island. The elastic constants of the $\mathrm{Si}_{.7} \mathrm{Ge}_{0.3}$ are $\mathrm{c}_{11}=153.9 \mathrm{GPa}, \mathrm{c}_{12}=58.9 \mathrm{GPa}$, and $\mathrm{c}_{44}=75.3 \mathrm{GPa}$, which are calculated by a linear interpolation between the elastic constants of $\mathrm{Si}$ and $\mathrm{Ge}[10,11]$.

Figure 3 shows the numerical results for the distributions of (a) the displacement in the $x$ direction, $\mathrm{u}$, and (b) the thickness strain, $\varepsilon_{\mathrm{zz}}$, along the $\mathrm{x}$-axis at $\mathrm{y}=0$ at different relaxation time. The displacement is normalized by $\varepsilon_{\mathrm{m}} \mathrm{L} / 2$, and the strain is normalized by the initial thickness 
strain, $2 \varepsilon_{\mathrm{m}} \mathrm{c}_{12} / \mathrm{c}_{11}$. Before relaxation, the displacement is zero and the normalized strain is 1 . When fully relaxed, the displacement is linear with the total expansion $\varepsilon_{\mathrm{m}} \mathrm{L}$, and the thickness strain becomes all zero. Figure 3 shows that the film is almost fully relaxed by time $t=0.5 t_{\mathrm{E}}$.
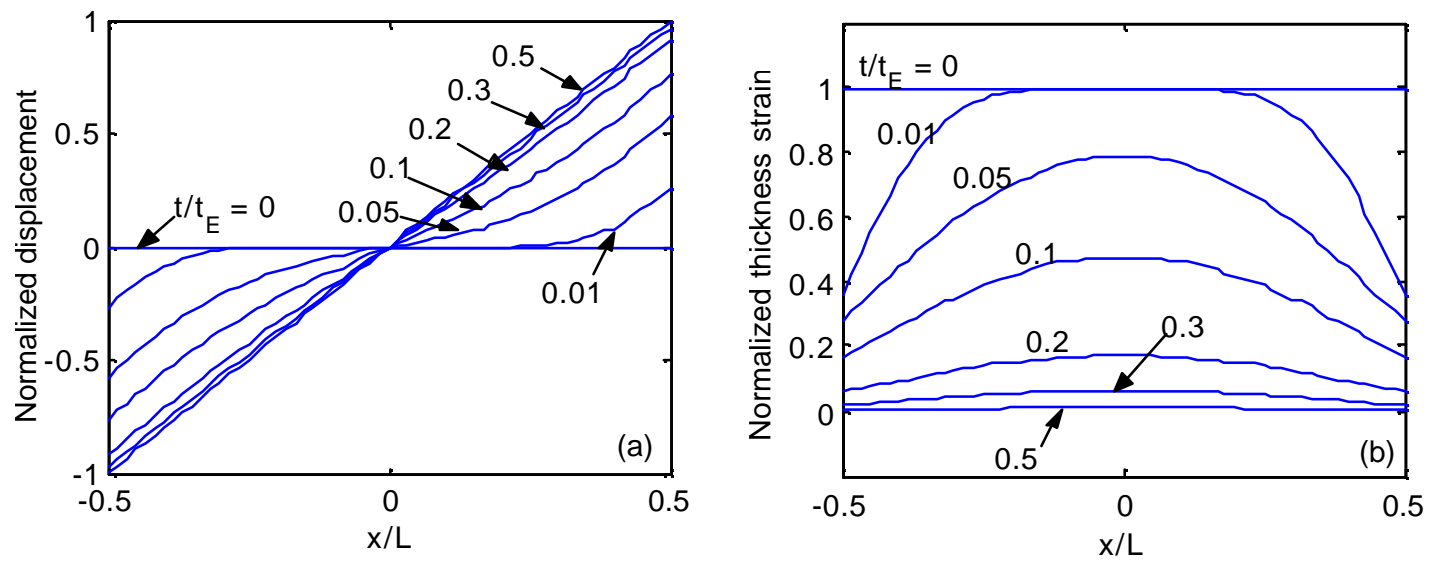

Figure 3. Numerical results: (a) Normalized displacement and (b) normalized thickness strain.

We calculate the average strain in the thickness direction of the film from the numerical results and compare with the experimental results obtained from the measured lattice constants through Eq. (1). Figure 4 shows the normalized average thickness strain, $\bar{\varepsilon}_{z z}(\mathrm{t}) / \bar{\varepsilon}_{z z}(0)$, as a function of the normalized relaxation time, $\mathrm{t} / \mathrm{t}_{\mathrm{E}}$. The solid line is from the model, and the circles are from experiments. While the theoretical curve is independent of the island size $\mathrm{L}$ or the coefficient $\mathrm{D}$, the experimental data have to be converted using specific values of $\mathrm{L}$ and $\mathrm{D}$. We chose $\mathrm{D}=4.3 \mu \mathrm{m}^{2} / \mathrm{min}$ to best fit the experimental data to the curve. From the value and the definition of $\mathrm{D}$ (Eq. 8), we can calculate the viscosity of the glass at the annealing temperature. Here, we obtain $\eta=12.5 \mathrm{GPa} \cdot \mathrm{s}$, which is about two magnitudes greater than the critical viscosity estimated by Hobart, et al. [3].

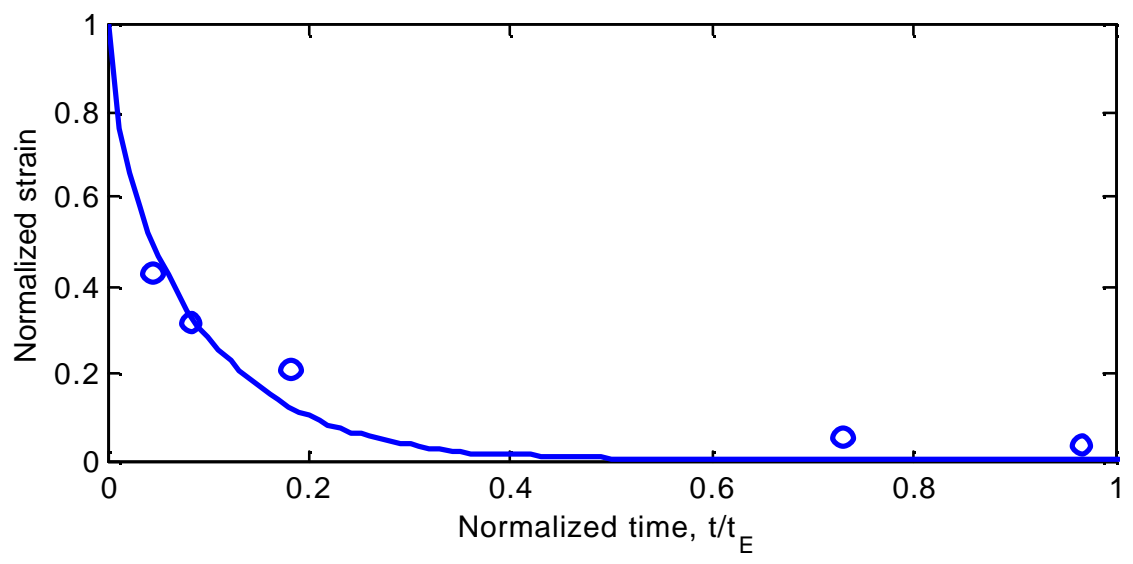

Figure 4. Comparison of the normalized average thickness strain as a function of the normalized relaxation time between the 2D model (solid line) and the experiments (circles). 


\section{EFFECT OF WRINKLING}

As observed in experiments, wrinkling occurs during relaxation, especially for large film islands. From the linear perturbation analysis [4,7], there exists a critical wave number, $\mathrm{k}_{\mathrm{c}}$, below which the wrinkle amplitude grows exponentially. The growth rate is independent of the film size. For an infinite film and a given wave number $\left(\mathrm{k}^{2} \mathrm{k}_{\mathrm{c}}\right)$, the wrinkle amplitude will saturate at an equilibrium value $[4,5,6]$. For finite size film islands, the inplane expansion competes with wrinkling [8]. As shown by Eq. (9), the time scale for inplane expansion is proportional to the island size. For small islands, inplane expansion is fast and wrinkles have no time to grow substantially. For large islands, it takes a long time for inplane expansion to reach the center, and significant wrinkles can grow near the center. Upon further annealing, the wrinkles may disappear when inplane expansion eventually reaches the center. Or, the wrinkles may become so severe that high tensile stress arises in the island, leading to fracture. The competition between wrinkling and inplane expansion sets a critical island size, below which the island relaxes without severe wrinkles [8].

\section{SUMMARY}

Both wrinkling and inplane expansion have been observed during relaxation of compressed SiGe film islands on a layer of borophosphorosilicate glass (BPSG). We formulate a 2D model for inplane expansion of small film islands and compare the results with the experiments. Wrinkling is ignored in the present model, but the qualitative effect is discussed.

\section{ACKNOWLEDGEMENTS}

This work is supported by NSF (CMS-9820713), by DARPA (N66001-00-1-8957), and by ARO (DAA655-98-1-0270). We thank D. J. Srolovitz and N. Sridhar for helpful discussions.

\section{REFERENCES}

1. K. Vanhollebeke, I. Moerman, P.Van Daele, and P. Demeester, Progress in Crystal Growth and Characterization of Materials 41, 1-55 (2000).

2. K. D. Hobart, F. J. Kub, M. Fatemi, M. E. Twigg, P. E. Thompson, T. S. Kuan, and C. K. Inoki, J. Electronic Materials 29, 897-900 (2000).

3. L. B. Freund and W. D. Nix (unpublished work).

4. N. Sridhar, D. J. Srolovitz, and Z. Suo, Appl. Phys. Lett. 78, 2482-2484 (2001)

5. N. Sridhar, D. J. Srolovitz, and B. N. Cox, submitted to Acta Materialia.

6. R. Huang and Z. Suo, Journal of Applied Physics, to appear in the February 12002 issue (Preprint available online at www.princeton.edu/ suo, Publication 120).

7. R. Huang and Z. Suo, International Journal of Solids and Structures, accepted for publication (Preprint available online at www.princeton.edu/ suo, Publication 121).

8. J. Liang, R. Huang, H. Yin, J. C. Sturm, K. D. Hobart, and Z. Suo, submitted to Acta Materialia (Preprint available online at www.princeton.edu/ suo, Publication 125).

9. M. Bruel, Electronics Letter 31, 1201-1202 (1995).

10. M. Neuberger, Group IV Semiconducting Materials, Handbook of Electronic Materials, vol. 5 (New York, IFI/Plenum, 1971).

11. C. Van De Walle and R. Martin, Phys. Rev. B 34, 5621-5634 (1986). 